\title{
Nonthermal irreversible electroporation for intracranial surgical applications
}

\author{
Laboratory investigation
}

\author{
Thomas L. Ellis, M.D., ${ }^{1}$ Paulo A. Garcia, B.S., ${ }^{2}$ John H. Rossmeisl Jr., D.V.M., M.S., ${ }^{3}$ \\ Natalia Henao-Guerrero, D.V.M., M.S., Diplom. A.C.V.A., ${ }^{3}$ \\ John Robertson, V.M.D., Ph.D., ${ }^{3}$ And Rafael V. Davalos, Ph.D. ${ }^{2,4}$ \\ ${ }^{1}$ Department of Neurosurgery, Wake Forest University School of Medicine, Winston-Salem, North \\ Carolina; ${ }^{2 B}$ Bioelectromechanical Systems Laboratory, School of Biomedical Engineering and Sciences, \\ Virginia Tech-Wake Forest University; ${ }^{3}$ Virginia-Maryland Regional College of Veterinary Medicine; \\ and ${ }^{4}$ Bioelectromechanical Systems Laboratory, Department of Engineering Science and Mechanics, Virginia \\ Polytechnic Institute and State University, Blacksburg, Virginia
}

\begin{abstract}
Object. Nonthermal irreversible electroporation (NTIRE) is a novel, minimally invasive technique to treat cancer, which is unique because of its nonthermal mechanism of tumor ablation. This paper evaluates the safety of an NTIRE procedure to lesion normal canine brain tissue.

Methods. The NTIRE procedure involved placing electrodes into a targeted area of brain in 3 dogs and delivering a series of short and intense electric pulses. The voltages of the pulses applied were varied between dogs. Another dog was used as a sham control. One additional dog was treated at an extreme voltage to determine the upper safety limits of the procedure. Ultrasonography was used at the time of the procedure to determine if the lesions could be visualized intraoperatively. The volumes of ablated tissue were then estimated on postprocedure MR imaging. Histological brain sections were then analyzed to evaluate the lesions produced.

Results. The animals tolerated the procedure with no apparent complications except for the animal that was treated at the upper voltage limit. The lesion volume appeared to decrease with decreasing voltage of applied pulses. Histological examination revealed cell death within the treated volume with a submillimeter transition zone between necrotic and normal brain.

Conclusions. The authors' results reveal that NTIRE at selected voltages can be safely administered in normal canine brain and that the volume of ablated tissue correlates with the voltage of the applied pulses. This preliminary study is the first step toward using NTIRE as a brain cancer treatment. (DOI: 10.3171/2010.5.JNS091448)
\end{abstract}

\section{KEY WoRDS - brain cancer therapy - minimally invasive surgery • nonthermal ablation $\quad$ tumor ablation $\quad$ electropermeabilization}

$\mathrm{T}$ HE destruction of tumors and other undesirable tissue through focal ablation has become an important, minimally invasive alternative to open surgery over the last several decades. Methods of tissue destruction include phototherapy, ${ }^{2}$ radiofrequency lesioning,${ }^{7,40}$ cryoablation,,$^{53}$ microwave ablation, ${ }^{41,52}$ high-intensity focused ultrasonography, ${ }^{19,32,55}$ and laser interstitial thermotherapy., 31,49 Mechanisms of tissue destruction include changes in local temperature resulting in coagulation necrosis $^{18,50}$ (radiofrequency lesioning, laser interstitial thermotherapy, and high-intensity focused ultrasonography) and cell membrane rupture ${ }^{18}$ (cryoablation), generation of free-radicals (phototherapy), and in situ tissue fixation (microwaves). ${ }^{41}$ Each of these techniques has selective advantages when used in the brain.

Abbreviation used in this paper: NTIRE $=$ nonthermal irreversible electroporation.
Electroporation is a technique in which electrical pulses are used to permeabilize tissue, either reversibly or irreversibly, through formation of nanoscale openings in cellular membranes. Reversible electroporation has been used for years to facilitate genetic modifications of cell function via delivery of plasmid DNA, mRNA, and macromolecules through the transient creation of nanopores within the cell membrane. ${ }^{16,42,45}$ Reversible electroporation can also be used to increase permeability to allow delivery of cytotoxic agents for targeted cell death in a process referred to as electrochemotherapy. ${ }^{12,23,24}$ When the electric field strength is above a critical value, irreversible electroporation occurs and results in cell death due to loss of homeostasis. ${ }^{17}$ Under most conditions, the irreversible increase in membrane permeability has been reported in conjunction with tissue thermal damage caused by electrical Joule heating. ${ }^{11,30}$ Joule heating is the dissipation of energy when an electrical current flows through a medium with resistance to that current. It is 
possible under certain conditions to permanently increase membrane permeability without thermal damage to the tissue $^{10}$ in a process referred to as NTIRE. This modality selectively disrupts cell membranes in non-CNS tissue, leading to the destruction of some, but not all tissues within the treated volume. ${ }^{10}$

The exact mechanism of electroporation is unknown. Measurements of changes in membrane electrical properties $^{25,27}$ and species transport across cellular membranes $^{37,38}$ reveal that electroporation allows otherwise impermeant molecules to diffuse more freely through membranes. Although the precise mechanism by which electrical pulses permeabilize membranes is not known, it is believed that the potential induced across the membrane leads to instability in the lipid bilayer, resulting in a localized alteration of membrane shape into aqueous pores through which molecules can pass. ${ }^{6,54}$ While reversible electroporation has been used for decades ${ }^{4,8,9,35,36,38,56}$ and knowledge of irreversible electroporation dates to the $1960 \mathrm{~s},{ }^{46}$ the use of NTIRE in vivo as a method of tissue ablation was only recently reported. ${ }^{15} \mathrm{~A}$ study in pig liver demonstrated that NTIRE protocols can be designed to destroy certain cells within the treated area while preserving major blood vessels and duct scaffolds. ${ }^{44} \mathrm{~A}$ study in dogs revealed that NTIRE can produce focal tissue ablation in the prostate while sparing the urethra. ${ }^{39}$

This study reports the first in vivo experimental use of NTIRE to ablate normal brain. Five purpose-bred canines were used. Three dogs were subjected to NTIRE therapy at differing voltage configurations. Two additional dogs were used as controls. In the fourth animal, 2 lesions were created to study the upper safety limit of the procedure. The fifth dog was used as a sham to isolate the tissue effects of electrode insertion without NTIRE treatment. The purposes of this study are to demonstrate that NTIRE at certain voltages can be used safely to create lesions within the brain, and there is a trend between applied voltage and the lesion volume.

\section{Methods}

The pilot study was approved by the Institutional Animal Care and Use Committee and performed in a Good Laboratory Practices-compliant facility. The dogs were systemically healthy and neurologically intact prior to the study, based on normal physical and neurological examinations, results of complete blood counts, and serum biochemistry profiles. No abnormalities were detected on scalp-recorded electroencephalograms and baseline MR imaging examinations of the brain. After administration of general anesthesia, a neuromuscular blockade, and an anticonvulsant (phenobarbital $6 \mathrm{mg} / \mathrm{kg}$ intravenously), routine craniectomies were performed to expose the right parietotemporal region of the brain of each dog. Focal ablative NTIRE lesions were created in the ectosylvian gyrus using blunt tip electrodes (Fig. 1) attached to the NanoKnife (AngioDynamics). This device is an electric pulse generator in which the desired NTIRE pulse parameters can be programmed prior to administering a treatment. The design allows the user to monitor the resulting current from the treatment and to automatically

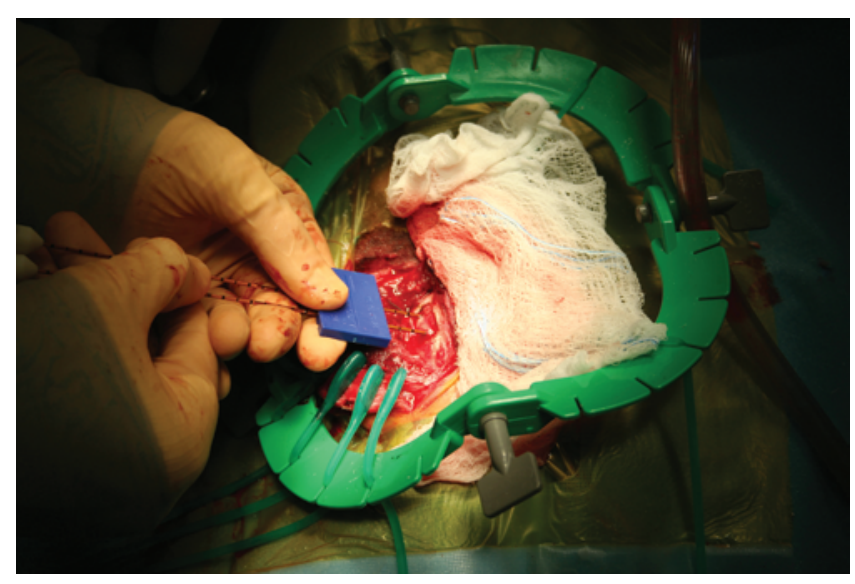

FIG. 1. Photograph showing the blunt-tip dual probe insertion during the intracranial NTIRE procedure.

suspend the delivery of the pulses if a current threshold is exceeded. The blunt tip electrodes are connected via a 6-ft insulated cable to the generator. The pulses are conducted from the generator to the tip of the electrodes after insertion into the tissue and upon activation of a foot pedal.

For Dog 1, a single probe with both an energized and grounded contact was placed at a depth of $2 \mathrm{~mm}$ below the gyral surface. For the remaining test animals, smaller dual probes (one energized and the other grounded) were placed at a depth of $7 \mathrm{~mm}$ below the gyral surface. The dual probes are similar in dimensions to those used in deep brain stimulation. The NTIRE lesions were created by administering 9 sets of 10 pulses at a repetition rate of $4 \mathrm{~Hz}$. The pulses were each $50 \mu \mathrm{sec}$ in duration and were configured with alternating polarity between each set to minimize total charge delivered to the brain. The pulse sets were delivered in 3.5-second intervals. The strength of the electric field applied is dependent on the voltage configuration between the energized and grounded electrodes. Values for each dog are given in Table 1. The voltage and pulse parameters were determined from the literature and from ex vivo experiments on canine brain.,15,29,39 Using these parameters, the charge delivered during this study was typical of that used in humans during electroconvulsive therapy. ${ }^{26}$ Dogs 1,2 , and 3 were treated with 1600,1000 , and $500 \mathrm{~V}$, respectively, to assess whether lower voltages could still produce neuronal ablation.

One control animal (Dog 4) was treated at a higher voltage to study the upper safety limit of the procedure. In this animal, 2 lesions were created using the dual electrode configuration at 1000 and $2000 \mathrm{~V}$. The last animal (Dog 5) was used as a sham control to examine the physical effects of electrode insertion without the NTIRE pulses. Nonenergized electrodes were advanced into the brain and were maintained in place for approximately 30 seconds, the time required to deliver the NTIRE treatments in the other animals.

During the NTIRE procedure, each animal underwent intraoperative ultrasonography to assess the position and extent of the lesion created. Postoperatively, all 
TABLE 1: Pulse parameters used in NTIRE brain treatment in dogs*

\begin{tabular}{|c|c|c|c|c|c|c|c|}
\hline Dog No. & $\begin{array}{l}\text { Blunt Tip Elec- } \\
\text { trodes }\end{array}$ & $\begin{array}{l}\text { Electrode Expo- } \\
\text { sure }(\mathrm{mm})\end{array}$ & $\begin{array}{c}\text { Separation } \\
\text { Distance }(\mathrm{mm})\end{array}$ & Voltage (V) & $\begin{array}{c}\text { Total } \\
\text { Energy }(\mathrm{J})\end{array}$ & Total No. of Pulses & $\begin{array}{l}\text { Pulse Duration } \\
\quad(\mu \mathrm{sec})\end{array}$ \\
\hline 1 & single & 7 & 8 & 1600 & 18.4 & 9 sets of 10 pulses & 50 \\
\hline 2 & dual & 5 & 5 & 1000 & 8.8 & 9 sets of 10 pulses & 50 \\
\hline 3 & dual & 5 & 5 & 500 & 0.8 & 9 sets of 10 pulses & 50 \\
\hline \multirow[t]{2}{*}{4 (control) } & dual & 5 & 5 & 1000 & 5.2 & 9 sets of 10 pulses & 50 \\
\hline & dual & 5 & 10 & 2000 & 39.4 & 9 sets of 10 pulses & 50 \\
\hline 5 (control) & dual & 5 & 5 & 0 & 0 & 0 & NA \\
\hline
\end{tabular}

* NA = not applicable.

animals underwent immediate MR imaging evaluation with and without contrast and recovered from anesthesia. Additional MR imaging was then performed 48 hours after the procedure on Dogs 2 and 3 to evaluate possible edema generation. Dogs were humanely killed 72 hours following the NTIRE procedure by intravenous barbiturate overdosage, and brain sections were submitted from all dogs for histological analysis.

After MR imaging, open-source image analysis software $($ OsiriX) was used to generate a $3 \mathrm{D}$ reconstruction from the traced DICOM images. ${ }^{21}$ Axial T2-weighted MR images were used for Dog 1, and T1-weighted with contrast were used for Dogs 2 and 3 to calculate the lesion volumes.

To verify that the NTIRE treatment was not associated with thermal damage, a thermal isoeffective dose analysis was used. ${ }^{48}$ Thermal isoeffective dose calculations are typically used for procedures involving complex variations in temperature as a function of time. Such calculations determine the amount of time it would take to equivalently damage the tissue as if it were held at a constant temperature. Typically $43^{\circ} \mathrm{C}$ is chosen due to the abundant experimental data at this temperature..$^{5,13,48}$ It has been shown that neuronal damage occurs if temperature is elevated to $43^{\circ} \mathrm{C}$ for 60 minutes $\left(t_{43}=60 \mathrm{~min}\right.$ utes). ${ }^{33}$ The following expression describes the duration necessary to hold the tissue at $43^{\circ} \mathrm{C}$ to result in thermal isoeffective dose:

$t_{43}=\int_{t=0}^{t=\text { final }} R^{\left(43-T_{t}\right)} d t$

where $T_{t}$ is the average temperature during the time interval $d t$ and $R$ is the number of minutes needed to compensate for a $1^{\circ} \mathrm{C}$ temperature change either above or below a breakpoint of $43^{\circ} \mathrm{C}$. $R$ is 0.25 when $T_{t} \leq 43^{\circ} \mathrm{C}$, and $R$ is 0.5 when $T_{t}>43^{\circ} \mathrm{C} .3^{33,48}$ The temperature distribution was calculated using similar methods to the ones described by Edd and Davalos ${ }^{14}$ with the physical properties from Garcia et al..$^{21}$

\section{Results}

After the procedure, the animals were evaluated and treated in the standard postcraniectomy fashion for dogs. There was no significant deterioration in neurological ability or coma scale scores from baseline evaluations. The animals were able to ambulate and eat within 10 hours of the procedure, suggesting that lesioning was well tolerated. No seizures were observed. Analysis of the intraoperative ultrasonography obtained for each animal revealed a clearly demarcated hypoechoic zone with hyperechoic rim within the targeted brain parenchyma (Fig. 2).

Magnetic resonance imaging examinations performed immediately postoperatively revealed fluid accumulation within the ablation sites and a focal disruption of the blood-brain barrier (Fig. 3). These images also show that the NTIRE ablation zones were sharply demarcated and iso- to hypointense on T1-weighted sequences, hyperintense on $\mathrm{T} 2$-weighted sequences, and peripherally contrast enhancing following intravenous administration of Gd. The 48-hour post-NTIRE MR imaging studies demonstrated edema in the subcortical white matter adjacent to the zone of ablation (Fig. 4). This edema was not associated with clinical effects in the dogs, and therefore it was not specifically treated.

The NTIRE lesion in the brain of Dog 1 was more superficial than the lesions in other animals, due to the electrode configuration used. In this animal, a single probe was inserted parallel to the surface of the brain at a

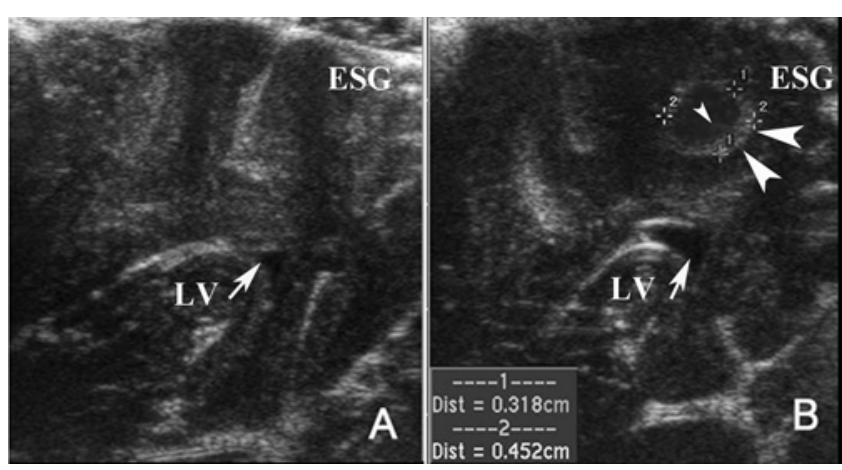

FIG. 2. Dog 1. Ultrasonographic images obtained intraoperatively but pre-NTIRE treatment (A) and 24 hours post-NTIRE treatment (B). The NTIRE ablation zone is clearly visible as a well-demarcated, hypoechoic circular lesion (small arrowhead) with a hyperechoic rim (large arrowheads). ESG = ectosylvian gyrus; LV = lateral ventricle. 

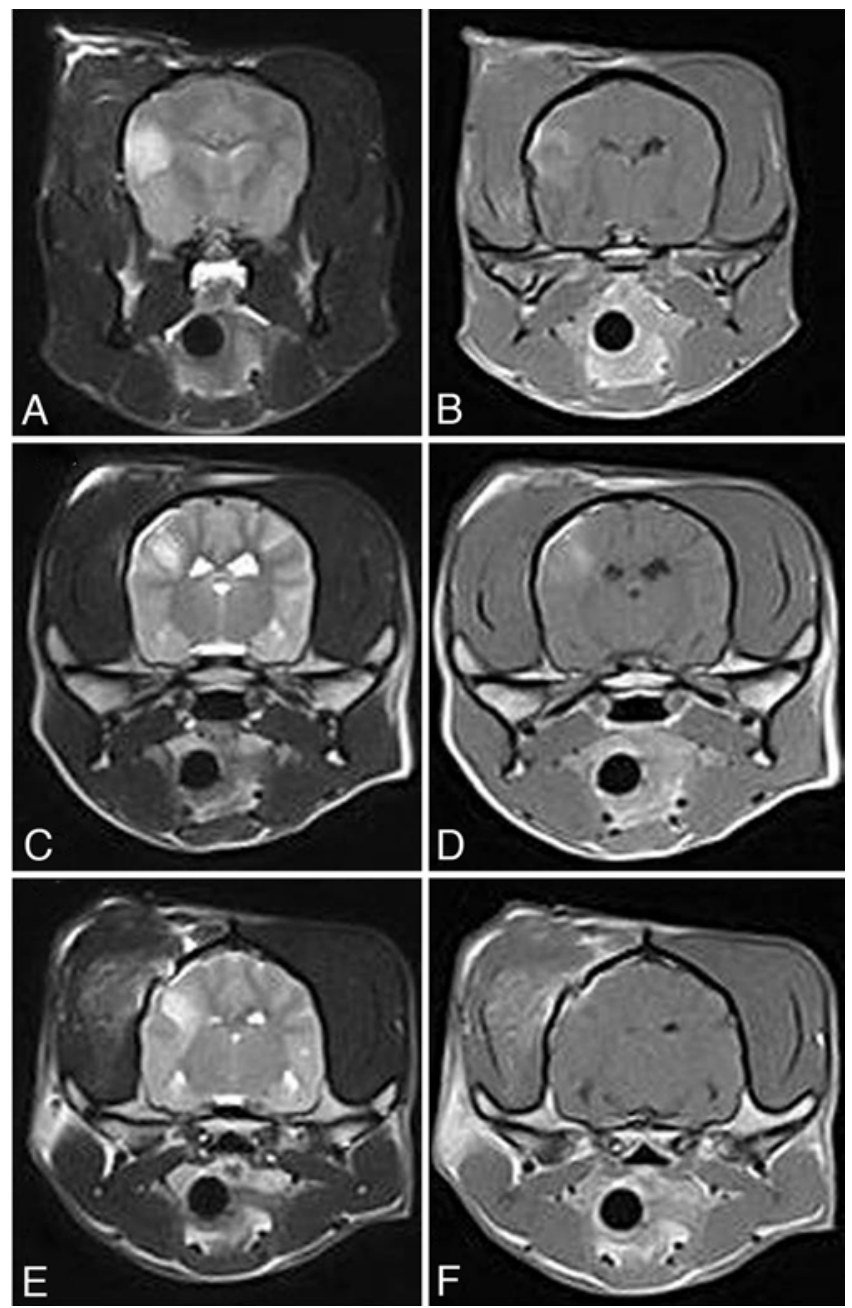

FIG. 3. Magnetic resonance images obtained immediately after the procedure in Dogs 1 (A and B, $1600 \mathrm{~V}, 8-\mathrm{mm}$ electrode separation), 2 (C and D, 1000 V, 5-mm electrode separation), and 3 (E and F, 500 V, 5-mm electrode separation). The left panels are T2-weighted images, and the right panels are T1-weighted contrast-enhanced images.

depth of $2 \mathrm{~mm}$. Grossly visible brain edema and surface blanching of the gyrus overlying the inserted electrode were observed within 2 minutes of completion of NTIRE procedure. This edema resolved completely following intravenous administration of $1.0 \mathrm{~g} / \mathrm{kg}$ of $20 \%$ mannitol. Because of these effects, the subsequent animals were treated with a smaller dual probe configuration with electrodes inserted perpendicular to the brain at a depth of $7 \mathrm{~mm}$. Brain edema and surface blanching were not observed during treatment of the remaining dogs.

The microscopic lesions from the histopathological analysis correlated well with the gross appearance and MR imaging sequences in Dogs 1-3. Histological comparison between the sham control and Dog 3 revealed that the isolated effect of electrode insertion is limited when compared with the NTIRE lesion (Fig. 5). Histopathological sections also demonstrated that the NTIRE lesions have a submillimeter line of demarcation between areas of necrosis and normal brain (Fig. 6A). The areas of treatment are represented by foci of malacia and dis-
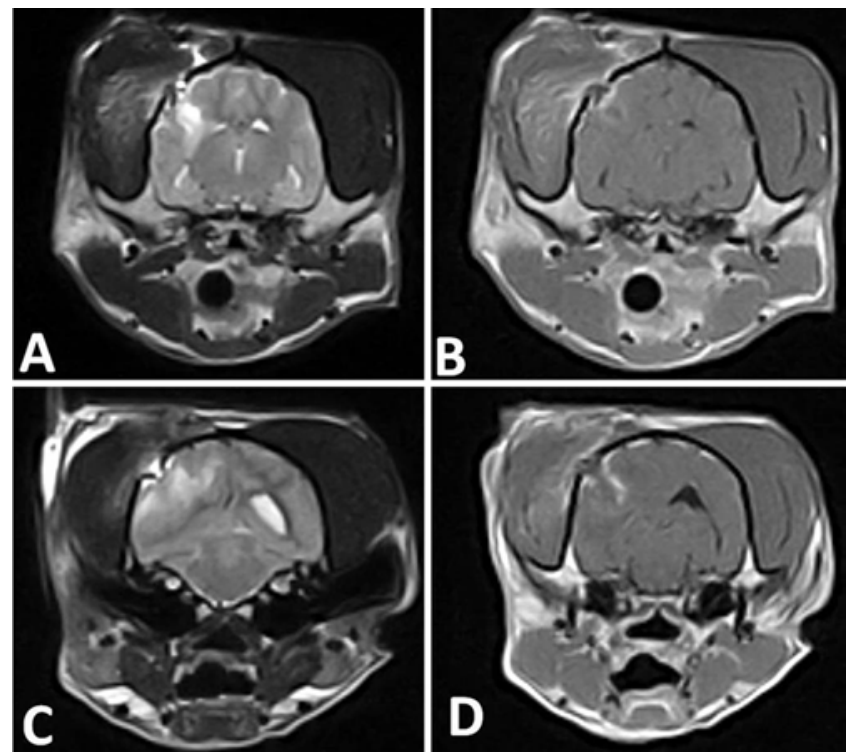

FIG. 4. Magnetic resonance images obtained 48 hours after the procedure in Dogs 3 (A and B, $500 \mathrm{~V}$ ) and 2 (C and D, $1000 \mathrm{~V})$. The left panels are T2-weighted MR images, and the right panels are T1weighted contrast-enhanced images. All images correspond to a 5-mm electrode separation.

sociation of white and gray matter. Small perivascular hemorrhages were present, although there appeared to be some sparing of major blood vessels (Fig. 6B). Highvoltage pulses in Dog 4 were associated with nonselective coagulative necrosis of all tissues within the treatment field (Fig. 6C), resulting in lacunar infarction due to arterial thrombosis. Moderate diffuse perivascular and intraglial edema, reactive gliosis, as well as death of neuronal and glial cells were also observed (Fig. 7). The treatment area was moderately infiltrated with mixed inflammatory cells, including neutrophils, macrophages, plasma cells, and small lymphocytes.

Smaller lesions were observed when decreasing the voltage between dogs. As a result, customizing the pulse parameters should allow the ablation of volumes of varying sizes and shapes. A maximum thermal isoeffective dose, $\mathrm{t}_{43}$, of 5.6, 2.0, and 0.5 minutes were calculated for Dogs 1,2, and 3, respectively (Table 2). The maximum $\mathrm{t}_{43}$ values were calculated at the electrode-tissue interface, where the highest electric field intensity is generated. Dog 4 experienced $\mathrm{at}_{43}$ for longer than 60 minutes in the areas surrounding the dual electrodes due to the high electric field in these regions. These results confirmed that ablation of the canine brain tissue in Dogs 1-3 resulted from irreversible cell membrane damage caused by NTIRE, rather than thermal damage.

\section{Discussion}

Nonthermal irreversible electroporation is a promising new technique for the ablation of tissue and tumors. ${ }^{1,15,29,39}$ A historical review by Rubinsky ${ }^{43}$ revealed that the first published reports documenting electroporation were written at the beginning of the 20 th century and 


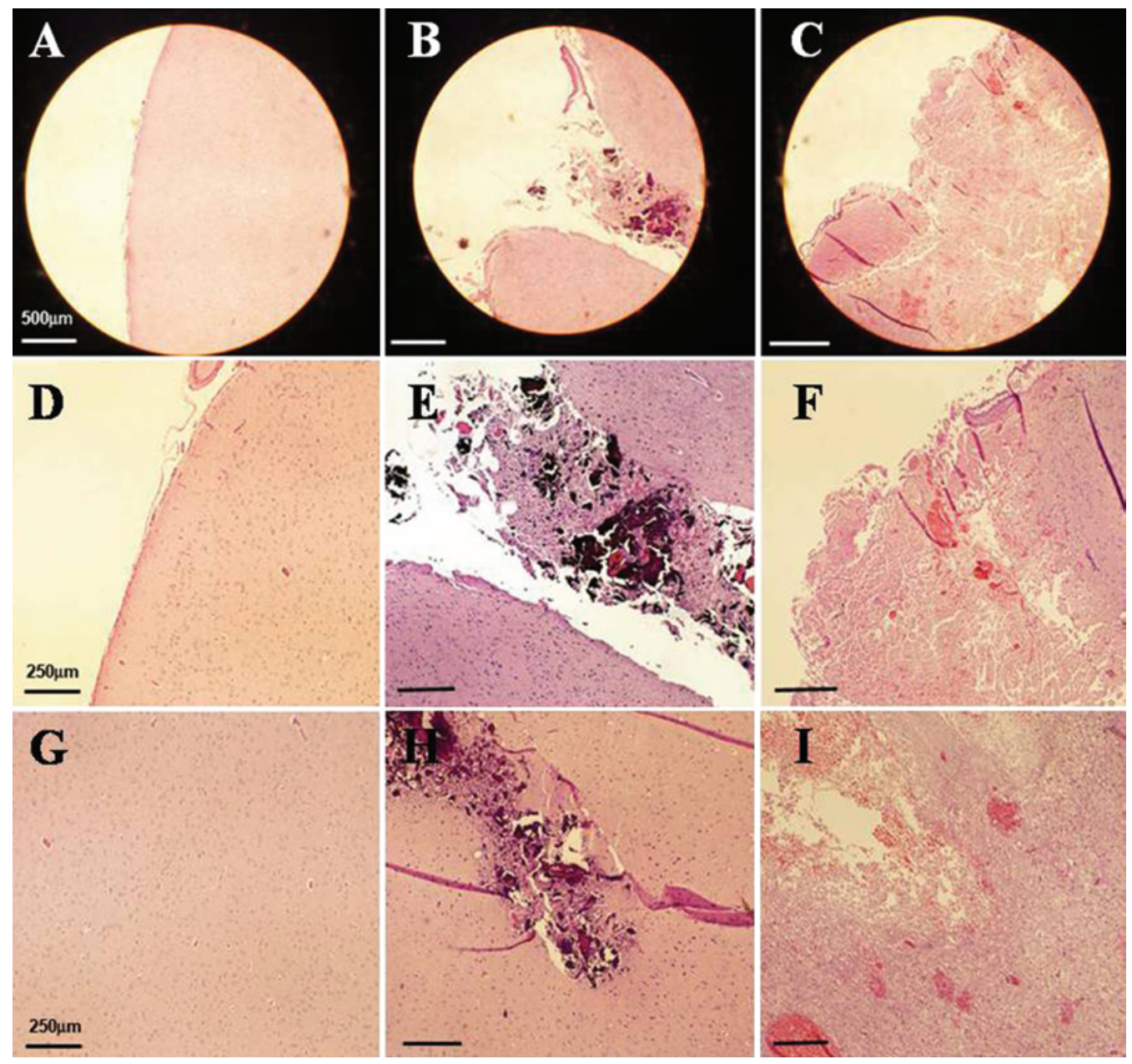

FIG. 5. Photomicrographs of normal canine brain at the surface (A and D) and deep (G) levels, sham study to isolate the mechanical effects of electrode insertion at the surface $(B$ and $E)$ and deep $(\mathbf{H})$ levels, and the NTIRE effect on brain at the surface (C and F) and deep (I) levels. H \& E, original magnification $\times 75(A-C)$, and $\times 150(D-I)$.

that the phenomenon may have been observed as early as the mid-18th century. The first methodical studies describing electroporation in amphibian nervous tissue arose in the 1950s. ${ }^{20,51}$ In a pivotal publication subsequent to this, Sale and Hamilton ${ }^{46}$ described the lethal effect of nonthermal electrical fields applied to cells and organisms in suspension. The ability of cellular membranes to recover from milder electrical pulses in what is now called "reversible electroporation" was first elucidated in the 1970s. ${ }^{4,22,28}$ In 1993, Salford et al ${ }^{47}$ first reported on the combined use of electropermeabilization and bleomycin in a rodent glioma model, demonstrating a doubling in survival time compared with animals treated with bleomycin alone.

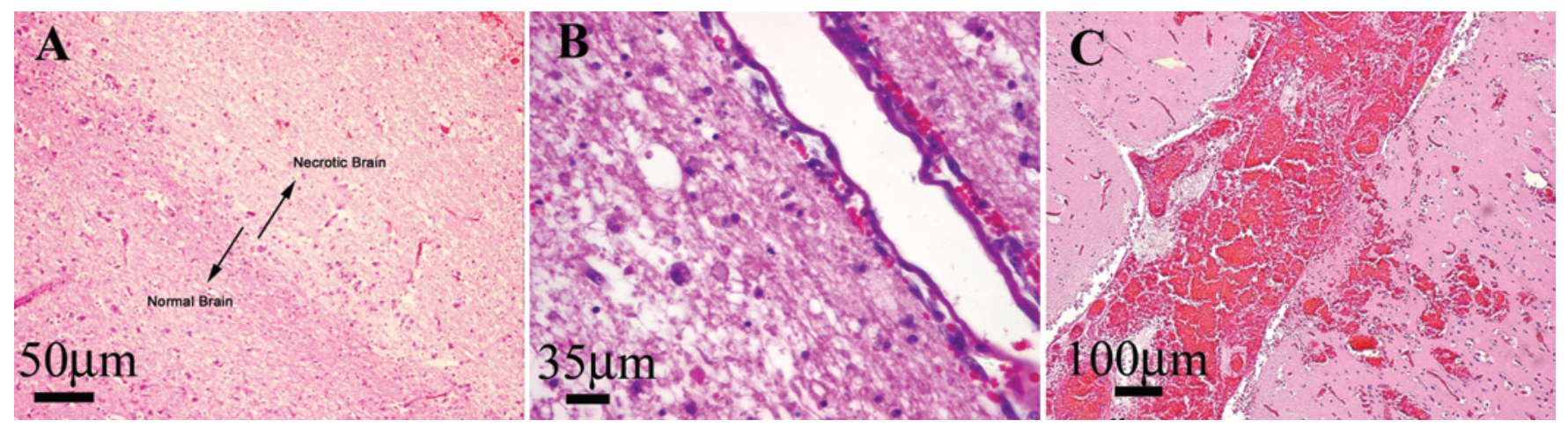

FIG. 6. Photomicrographs of NTIRE-treated brains. In Dog 2, a very sharp transition zone between necrotic and normal tissue (A) and sparing of major blood vessels (B) are shown. In control (Dog 4), a thrombus was detected (C). H \& E. 


\section{T. L. Ellis et al.}

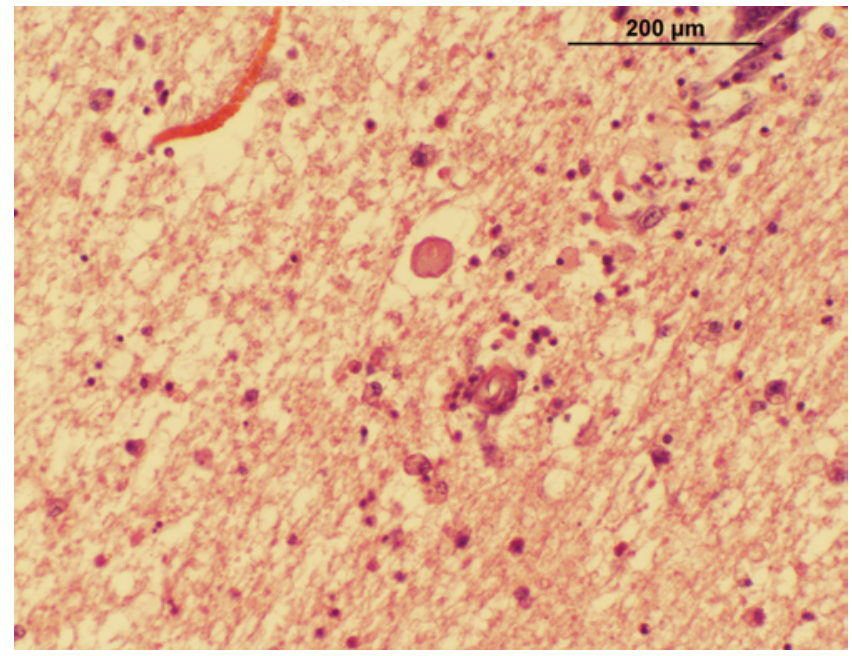

FIG. 7. Photomicrograph. In this section of brain, taken at the site of NTIRE treatment, there is moderate diffuse perivascular and intraglial edema, as well as death of neuronal and glial cells. Modest reactive gliosis is seen. The treatment area is infiltrated with mixed inflammatory cells, including neutrophils, macrophages, plasma cells, and small lymphocytes. This section is representative of the NTIRE treatment area. $\mathrm{H}$ $\& \mathrm{E}$, original magnification $\times 40$.

To irreversibly electroporate cell membranes, the electric field in the targeted region needs to be above a critical value, which is dependent on a variety of conditions such as tissue type and pulse parameters (amplitude, duration, frequency, and number).${ }^{14}$ Under most conditions, the electric field required to produce irreversible electroporation is associated with significant tissue heating and thermal damage. However, Davalos et al. ${ }^{10}$ demonstrated that certain pulse parameters can produce irreversible electroporation without thermal damage, and that NTIRE can be used to destroy substantial volumes of tissue in vivo. Since then, researchers have demonstrated the use of NTIRE in small and large animal models in the liver ${ }^{15,29}$ and prostate, ${ }^{39}$ and on implanted mouse sarcomas. ${ }^{1}$ Because of the nonthermal mechanism, NTIRE can preserve the extracellular matrix, axons, and major blood vessels in non-CNS tissues..$^{39,43}$ Furthermore, the procedure can be monitored in real time using ultrasonography and confirmed with ultrasonography and MR imaging. ${ }^{29,30,44}$ In NTIRE studies outside the CNS, ablation is rapid in onset and resolution, allowing early repopulation of the ablated region with healthy cells. ${ }^{39,43}$ Although treatment success is not dependent on the immune system, a tumor-specific immune response appears to occur. ${ }^{39}$

This paper describes the first systematic in vivo study of NTIRE for intracranial surgery. Focal lesions were created in the right parietotemporal lobe of dogs using blunt tip probes. The ablation was confirmed with histopathological analysis, revealing a submillimeter boundary between the necrotic and normal brain. Reconstructed lesion volumes of $1.655 \pm 0.255,0.599 \pm 0.081$, and $0.258 \pm 0.027 \mathrm{~cm}^{3}$ were calculated from the postoperative MR images. The accuracy of the computed lesion volumes was limited to the interval between the MR images $(2.5-3.0 \mathrm{~mm})$. Pulse durations $(50 \mu \mathrm{sec})$ shorter than
TABLE 2: Computations for each NTIRE-treated dog

\begin{tabular}{cccc}
\hline Dog No. & Voltage $(\mathrm{V})$ & Lesion Vol $\left(\mathrm{cm}^{3}\right)$ & $\begin{array}{c}\text { Thermal Isoeffective Dose } \\
(\mathrm{mins})^{*}\end{array}$ \\
\hline 1 & 1600 & $1.655 \pm 0.255$ & 5.6 \\
2 & 1000 & $0.599 \pm 0.081$ & 2.0 \\
3 & 500 & $0.258 \pm 0.027$ & 0.5 \\
\hline
\end{tabular}

* Neuronal damage occurs if the thermal isoeffective dose is $60 \mathrm{~min}$ utes according to Matsumi et al.

those described in previous studies were used to reduce the charge delivered to the tissue and to minimize the heating generated by the procedure. .15, $29,39^{-10}$

Nonthermal irreversible electroporation treatments are dependent on the tissue of interest, and the pulse parameters need to be selected correctly for complete focal ablation. Tissue heterogeneities (gray vs white matter) are of particular interest in the application of NTIRE for brain cancer, and future modeling studies will incorporate these effects on the resulting lesion volumes. Future work will also investigate the effects of NTIRE in different brain tissue states (for example, healthy vs tumor tissue) and brain tumor types, in the effort to enhance tissue selectivity. In addition, we plan to investigate the effect of pulse number and frequency on the NTIRE lesion volume. At this point, it is unclear whether 1 application of NTIRE pulses is sufficient for a successful treatment or if multiple applications are needed to cover a volume of interest. Numerical models will have to be developed to establish the parameters needed to predict manageable NTIRE lesion volumes. At that point, this technology may be applicable for intracranial surgical applications in which ablation of a defined volume of brain parenchyma is desired.

Our results support the hypothesis that NTIRE can be used safely in the brain and that lesion volume can be correlated with applied voltage. Future studies in an experimental rodent population will focus on the use of NTIRE for the ablation of brain tumors. In this study, as in other studies of soft-tissue organs, NTIRE-associated edema developed following treatment. Although the vasogenic edema observed on the MR imaging examinations in the dogs in this study was not associated with any clinical deterioration, it is a cause of major concern. Brain edema after NTIRE should be anticipated and treated with perioperative corticosteroids.

Although the results presented are preliminary, NTIRE may offer an advantage over resection for selected brain tumors. The small electrode size makes the procedure minimally invasive and adaptable to virtually any neuroanatomical location with existing stereotactic guidance systems. Nonthermal irreversible electroporation creates a sharply delineated volume of ablated tissue with submillimeter resolution that may make it suitable for treating deep-seated, well-circumscribed brain tumors. The minimal heat generation during treatment and sparing of major blood vessels may also make it appropriate for tumors adjacent to or those enveloping critical vascular structures. 


\section{Nonthermal irreversible electroporation for intracranial surgery}

In studies outside the nervous system, NTIRE produces a penumbra of nondestructive increase in membrane permeability beyond the zone of tissue ablation. ${ }^{10,34}$ It is unclear at this time whether this phenomenon occurs in the brain or in brain tumors. Future efforts within our group will explore this question to determine if the delivery of chemotherapy or other cytotoxic agents can be enhanced in reversibly electroporated areas of infiltrative brain tumors. Determining the extent of any reversible electroporation as well as the minimal electric field threshold necessary to achieve it will be critical for development of a paradigm to plan and treat brain tumors, especially high-grade gliomas.

\section{Conclusions}

This study is the first to examine the safety of NTIRE in the brain. Procedures were conducted in the brain of 3 canines using minimally invasive electrodes. It was observed that NTIRE can be safely administered and that the volume of ablated tissue correlates with the voltage of the applied electric pulses. This preliminary study is the first step toward using NTIRE as a focal ablation treatment for brain cancer and other intracranial disorders.

\section{Disclosure}

This work was supported in part by the Coulter Foundation. In addition, AngioDynamics loaned the NanoKnife unit used in the study. Drs. Rossmeisl and Robertson and Mr. Garcia are patent holders of "Irreversible Electroporation to Treat Aberrant Masses." Dr. Davalos has pending patents in the area of irreversible electroporation and provides minimal consulting in the area of numerical modeling of electric fields, received financial support of nonstudyrelated research by AngioDynamics, and serves as a consultant for AngioDynamics.

Author contributions to the study and manuscript preparation include the following Conception and design: Davalos, Garcia, Rossmeisl, Robertson. Acquisition of data: all authors. Analysis and interpretation of data: Davalos, Ellis, Garcia, Rossmeisl, HenaoGuerrero. Drafting the article: Davalos, Ellis, Garcia, Rossmeisl, Robertson. Critically revising the article: all authors. Reviewed final version of the manuscript and approved it for submission: all authors. Study supervision: Davalos, Garcia, Rossmeisl, Robertson.

\section{Acknowledgments}

The authors thank Gregory B. Daniel, Carolina Ricco, Dana Calicott, Barbara Kafka, and Stephanie Milburn for their assistance in surgery and Lindsey Buracker, Bobby Neal, and Chris Arena for their help in treatment planning.

\section{References}

1. Al-Sakere B, André F, Bernat C, Connault E, Opolon P, Davalos RV, et al: Tumor ablation with irreversible electroporation. PLoS ONE 2:e1135, 2007

2. Al-Waili NS, Butler GJ: Phototherapy and malignancy: possible enhancement by iron administration and hyperbaric oxygen. Med Hypotheses 67:1148-1158, 2006

3. Atsumi H, Matsumae M, Kaneda M, Muro I, Mamata Y, Komiya T, et al: Novel laser system and laser irradiation method reduced the risk of carbonization during laser interstitial thermotherapy: assessed by MR temperature measurement. Lasers Surg Med 29:108-117, 2001
4. Baker PF, Knight DE: Calcium-dependent exocytosis in bovine adrenal medullary cells with leaky plasma membranes. Nature 276:620-622, 1978

5. Becker SM, Kuznetsov AV: Numerical modeling of in vivo plate electroporation thermal dose assessment. J Biomech Eng 128:76-84, 2006

6. Chizmadzhev YA, Zarnitsin VG, Weaver JC, Potts RO: Mechanism of electroinduced ionic species transport through a multilamellar lipid system. Biophys J 68:749-765, 1995

7. Cosman ER, Nashold BS, Bedenbaugh P: Stereotactic radiofrequency lesion making. Appl Neurophysiol 46:160-166, 1983

8. Coster HGA: A quantitative analysis of the voltage-current relationships of fixed charge membranes and the associated property of "punch-through". Biophys J 5:669-686, 1965

9. Crowley JM: Electrical breakdown of bimolecular lipid membranes as an electromechanical instability. Biophys J 13:711724,1973

10. Davalos RV, Mir IL, Rubinsky B: Tissue ablation with irreversible electroporation. Ann Biomed Eng 33:223-231, 2005

11. Davalos RV, Rubinsky B, Mir LM: Theoretical analysis of the thermal effects during in vivo tissue electroporation. Bioelectrochemistry 61:99-107, 2003

12. Dev SB, Hofmann GA: Electrochemotherapy-a novel method of cancer treatment. Cancer Treat Rev 20:105-115, 1994

13. Dewhirst MW, Viglianti BL, Lora-Michiels M, Hanson M, Hoopes PJ: Basic principles of thermal dosimetry and thermal thresholds for tissue damage from hyperthermia. Int J Hyperthermia 19:267-294, 2003

14. Edd JF, Davalos RV: Mathematical modeling of irreversible electroporation for treatment planning. Technol Cancer Res Treat 6:275-286, 2007

15. Edd JF, Horowitz L, Davalos RV, Mir LM, Rubinsky B: In vivo results of a new focal tissue ablation technique: irreversible electroporation. IEEE Trans Biomed Eng 53:1409-1415, 2006

16. Escoffre JM, Portet T, Wasungu L, Teissié J, Dean D, Rols MP: What is (still not) known of the mechanism by which electroporation mediates gene transfer and expression in cells and tissues. Mol Biotechnol 41:286-295, 2009

17. Esser AT, Smith KC, Gowrishankar TR, Weaver JC: Towards solid tumor treatment by irreversible electroporation: intrinsic redistribution of fields and currents in tissue. Technol Cancer Res Treat 6:261-274, 2007

18. Finelli A, Rewcastle JC, Jewett MA: Cryotherapy and radiofrequency ablation: pathophysiologic basis and laboratory studies. Curr Opin Urol 13:187-191, 2003

19. Foster RS, Bihrle R, Sanghvi NT, Fry FJ, Donohue JP: Highintensity focused ultrasound in the treatment of prostatic disease. Eur Urol 23 (1 Suppl):29-33, 1993

20. Frankenhaeuser B, Widen L: Anode break excitation in desheathed frog nerve. J Physiol 131:243-247, 1956

21. Garcia PA, Rossmeisl JH Jr, Robertson J, Ellis TL, Davalos RV: Pilot study of irreversible electroporation for intracranial surgery. Conf Proc IEEE Eng Med Biol Soc 2009:65136516, 2009 (Abstract)

22. Gauger B, Bentrup FW: A study of dielectric membrane breakdown in the Fucus egg. J Membr Biol 48:249-264, 1979

23. Glass LF, Pepine ML, Fenske NA, Jaroszeski M, Reintgen DS, Heller R: Bleomycin-mediated electrochemotherapy of metastatic melanoma. Arch Dermatol 132:1353-1357, 1996

24. Heller R, Gilbert R, Jaroszeski MJ: Clinical applications of electrochemotherapy. Adv Drug Deliv Rev 35:119-129, 1999

25. Hibino M, Itoh H, Kinosita K Jr: Time courses of cell electroporation as revealed by submicrosecond imaging of transmembrane potential. Biophys J 64:1789-1800, 1993

26. Jaffe R: The Practice of Electroconvulsive Therapy: Recommendations for Treatment, Training, and Privileging: A Task 
Force Report of the American Psychiatric Association, 2nd ed. Am J Psychiatry 159:331, 2002 (Book review)

27. Kinosita K Jr, Ashikawa I, Saita N, Yoshimura H, Itoh H, Nagayama K, et al: Electroporation of cell membrane visualized under a pulsed-laser fluorescence microscope. Biophys J 53:1015-1019, 1988

28. Kinosita K Jr, Tsong TY: Formation and resealing of pores of controlled sizes in human erythrocyte membrane. Nature 268:438-441, 1977

29. Lee EW, Loh CT, Kee ST: Imaging guided percutaneous irreversible electroporation: ultrasound and immunohistological correlation. Technol Cancer Res Treat 6:287-294, 2007

30. Lee RC, Zhang D, Hannig J: Biophysical injury mechanisms in electrical shock trauma. Annu Rev Biomed Eng 2:477509,2000

31. Leonardi MA, Lumenta CB, Gumprecht HK, von Einsiedel GH, Wilhelm T: Stereotactic guided laser-induced interstitial thermotherapy (SLITT) in gliomas with intraoperative morphologic monitoring in an open MR-unit. Minim Invasive Neurosurg 44:37-42, 2001

32. Lynn JG, Zwemer RL, Chick AJ: The Biological Application of Focused Ultrasonic Waves. Science 96:119-120, 1942

33. Matsumi N, Matsumoto K, Mishima N, Moriyama E, Furuta T, Nishimoto A, et al: Thermal damage threshold of brain tissue-histological study of heated normal monkey brains. Neurol Med Chir (Tokyo) 34:209-215, 1994

34. Miklavcic D, Semrov D, Mekid H, Mir LM: A validated model of in vivo electric field distribution in tissues for electrochemotherapy and for DNA electrotransfer for gene therapy. Biochim Biophys Acta 1523:73-83, 2000

35. Mir LM: Therapeutic perspectives of in vivo cell electropermeabilization. Bioelectrochemistry 53:1-10, 2001

36. Mir LM, Orlowski S, Belehradek J Jr, Paoletti C: Electrochemotherapy potentiation of antitumour effect of bleomycin by local electric pulses. Eur J Cancer 27:68-72, 1991

37. Neumann E, Kakorin S, Toensing K: Membrane electroporation and electromechanical deformation of vesicles and cells. Faraday Discuss 111:111-125, 137-157, 1998

38. Neumann E, Rosenheck K: Permeability changes induced by electric impulses in vesicular membranes. J Membr Biol 10:279-290, 1972

39. Onik G, Mikus P, Rubinsky B: Irreversible electroporation: implications for prostate ablation. Technol Cancer Res Treat 6:295-300, 2007

40. Organ LW: Electrophysiologic principles of radiofrequency lesion making. Appl Neurophysiol 39:69-76, 1976-1977

41. Ozaki T, Tabuse K, Tsuji T, Nakamura Y, Kakudo K, Mori I: Microwave cell death: Enzyme histochemical evaluation for metastatic carcinoma of the liver. Pathol Int 53:837-845, 2003

42. Parham JH, Iannone MA, Overton LK, Hutchins JT: Optimization of transient gene expression in mammalian cells and potential for scale-up using flow electroporation. Cytotechnology 28:147-155, 1998

43. Rubinsky B: Irreversible electroporation in medicine. Technol Cancer Res Treat 6:255-260, 2007
44. Rubinsky B, Onik G, Mikus P: Irreversible electroporation: a new ablation modality-clinical implications. Technol Cancer Res Treat 6:37-48, 2007

45. Ruby KM, Zheng B: Gene targeting in a HUES line of human embryonic stem cells via electroporation. Stem Cells 27:1496-1506, 2009

46. Sale AJ, Hamilton WA: Effects of high electric fields on micro-organisms. 3. Lysis of erythrocytes and protoplasts. Biochim Biophys Acta 163:37-43, 1968

47. Salford LG, Persson BR, Brun A, Ceberg CP, Kongstad PC, Mir LM: A new brain tumour therapy combining bleomycin with in vivo electropermeabilization. Biochem Biophys Res Commun 194:938-943, 1993

48. Sapareto SA, Dewey WC: Thermal dose determination in cancer therapy. Int J Radiat Oncol Biol Phys 10:787-800, 1984

49. Schwarzmaier HJ, Eickmeyer F, von Tempelhoff W, Fiedler VU, Niehoff H, Ulrich SD, et al: MR-guided laser-induced interstitial thermotherapy of recurrent glioblastoma multiforme: preliminary results in 16 patients. Eur J Radiol 59:208-215, 2006

50. Solbiati L, Ierace T, Goldberg SN, Sironi S, Livraghi T, Fiocca $\mathrm{R}$, et al: Percutaneous US-guided radio-frequency tissue ablation of liver metastases: treatment and follow-up in 16 patients. Radiology 202:195-203, 1997

51. Stampfli R, Willi M: Membrane potential of a Ranvier node measured after electrical destruction of its membrane. Experientia 13:297-298, 1957

52. Tabuse K: A new operative procedure of hepatic surgery using a microwave tissue coagulator. Nippon Geka Hokan 48: 160-172, 1979

53. Tacke J: Thermal therapies in interventional MR imaging. Cryotherapy. Neuroimaging Clin N Am 11:759-765, 2001

54. Weaver JC: Electroporation: a general phenomenon for manipulating cells and tissues. J Cell Biochem 51:426-435, 1993

55. Yang R, Sanghvi NT, Rescorla FJ, Galliani CA, Fry FJ, Griffith SL, et al: Extracorporeal liver ablation using sonographyguided high-intensity focused ultrasound. Invest Radiol 27: 796-803, 1992

56. Zimmermann U, Pilwat G, Riemann F: Dielectric breakdown of cell membranes. Biophys J 14:881-899, 1974

Manuscript submitted October 18, 2009.

Accepted May 18, 2010

Portions of the findings from Dog 1 were presented in abstract form as proceedings at the 31st Annual International Conference of the IEEE EMBS, Minneapolis, Minnesota, September 2-6, 2009.

Please include this information when citing this paper: published online June 18, 2010; DOI: 10.3171/2010.5 JNS091448.

Address correspondence to: Rafael V. Davalos, Ph.D., School of Biomedical Engineering and Sciences, Virginia Tech, 329 ICTAS Building, Stanger Street (MC 0298), Blacksburg, Virginia 24061. email: davalos@vt.edu. 